\title{
Validación del nombre Abies × masjoannis (Pinaceae)
}

\author{
P. Pablo Ferrer-Gallego ${ }^{1,2}$ Emilio Laguna', Diodoro Soto García ${ }^{3}$, Juan I. García Viñas ${ }^{4}$ \\ \& Emilio Pérez Bujarrabal ${ }^{5}$
}

'Servicio de Vida Silvestre. Centro para la Investigación y Experimentación Forestal, Generalitat Valenciana, Avda. Comarques del País Valencià 114, 46930 Quart de Poblet, España

${ }^{2}$ VAERSA. Avda. Cortes Valencianas 20, 46015 València, España

${ }^{3} \mathrm{C} /$ Romero Robledo, 13. 28008 Madrid. España

${ }^{4}$ Dto. Sistemas y Recursos Forestales, ETSI Montes Forestal y del Medio Natural, Ciudad Universitaria, s/n. 28040 Madrid. España

${ }^{5}$ Avda. Juan Pablo II, 64. 50009 Zaragoza. España

\section{Correspondencia \\ P.P. Ferrer-Gallego}

e-mail: flora.cief@gva.es

Recibido: 27 marzo 2017

Aceptado: 11 diciembre 2017

Publicado on-line: diciembre 2017

\begin{abstract}
Resumen
La denominación "Abies $\times$ masjoannis" se aplica a un híbrido entre Abies alba y A. pinsapo (Pinaceae). Sin embargo, no fue indicado ningún tipo nomenclatural para esta denominación y en consecuencia no puede ser considerado como un nombre válidamente publicado de acuerdo al Código Internacional de Nomenclatura o Código de Melbourne de 2012 (Art. 40.1 y H. 10.1). En la presente comunicación se valida el nombre.
\end{abstract}

Palabras clave: Abies, híbrido, nomenclatura, notomorfa.

\begin{abstract}
Validation of the name Abies $\times$ masjoannis (Pinaceae).

"Abies $\times$ masjoannis" is applied to a hybrid between Abies alba and $A$. pinsapo (Pinaceae). However, no type was indicated for "Abies $\times$ masjoannis" and therefore cannot be considered as a validly published name according to the International Code of Nomenclature or Melbourne Code, 2012 (Art. 40.1 and H. 10.1). In the present contribution it is validated.
\end{abstract}

Key words: Abies, hybrid, nomenclature, nothomorph.

\section{Introducción}

La denominación "Abies $\times$ masjoannis" se aplica a un híbrido entre Abies alba Mill. y A. pinsapo Boiss. (Pinaceae). Esta planta fue descrita a partir de varios ejemplares que crecen en jardines de la ciudad de Madrid y en otras provincias españolas, y también del estudio de la descendencia del cruzamiento entre los progenitores presentes en la finca Masjoan del arboricultor J. Masferrer, en término de Espinelves (Girona), lugar donde se cultivó y se difundió de manera abundante la primera generación filial (Soto García et al., 2004, 2013; Ruiz de la Torre, 2006). En el trabajo original, basado en dos recolecciones de diferente procedencia, una de 140 ejemplares procedentes de Masjoan y otra de 60 procedentes de Rocal (Navarra), con individuos juveniles y productores de piñas y siempre de origen verificado en semillas de piñas de $A$. pinsapo, se describe "Abies xmasjoannis" aportando detalladas características de diagnosis y diferenciando dentro de su variabilidad fenotípica tres notomorfas, denominadas: "alboides", "mesoides" y "pinsapoides", según el grado de semejanza con cada uno de los progenitores, dando a entender que el taxon con nombre infraespecífico y epíteto final "mesoides", que muestra las características intermedias entre sus dos progenitores (Soto García et al., 2004; Ruiz de la Torre, 2006) incluiría el tipo del nombre del taxon 


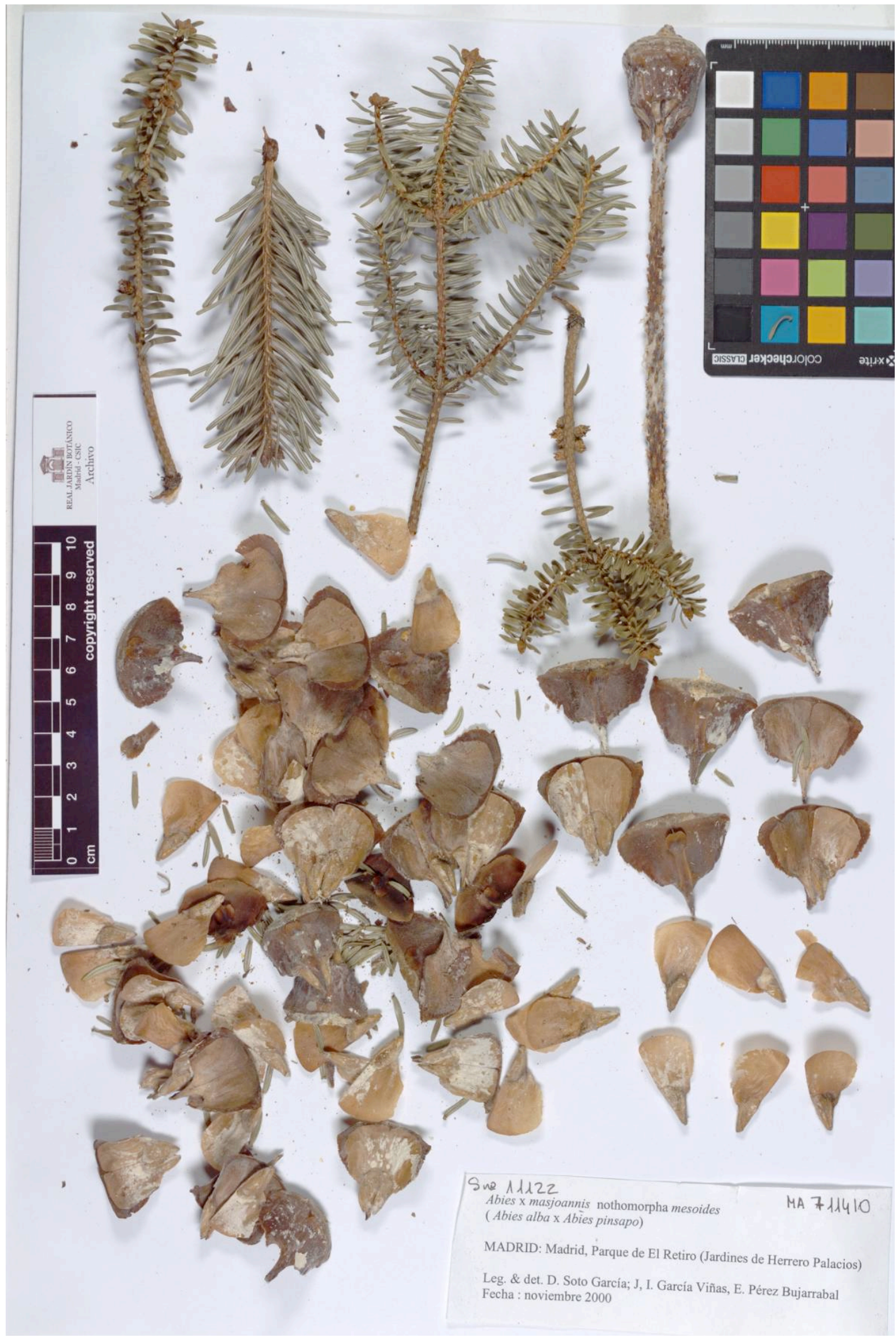

Figura 1. Holotipo de Abies xmasjoannis D. Soto, J.I. García Viñas et E.P. Bujarrabal [A. alba Mill. $\times$ A. pinsapo Boiss.] (MA 711410). Imagen reproducida con permiso del herbario MA. Figure 1. Holotype of Abies $\times$ masjoannis $D$. Soto, J.I. Garcia Viñas et E.P. Bujarrabal [A. alba Mill. x A. pinsapo Boiss.] (MA 711410). Courtesy of the herbarium MA; reproduced with permission. 


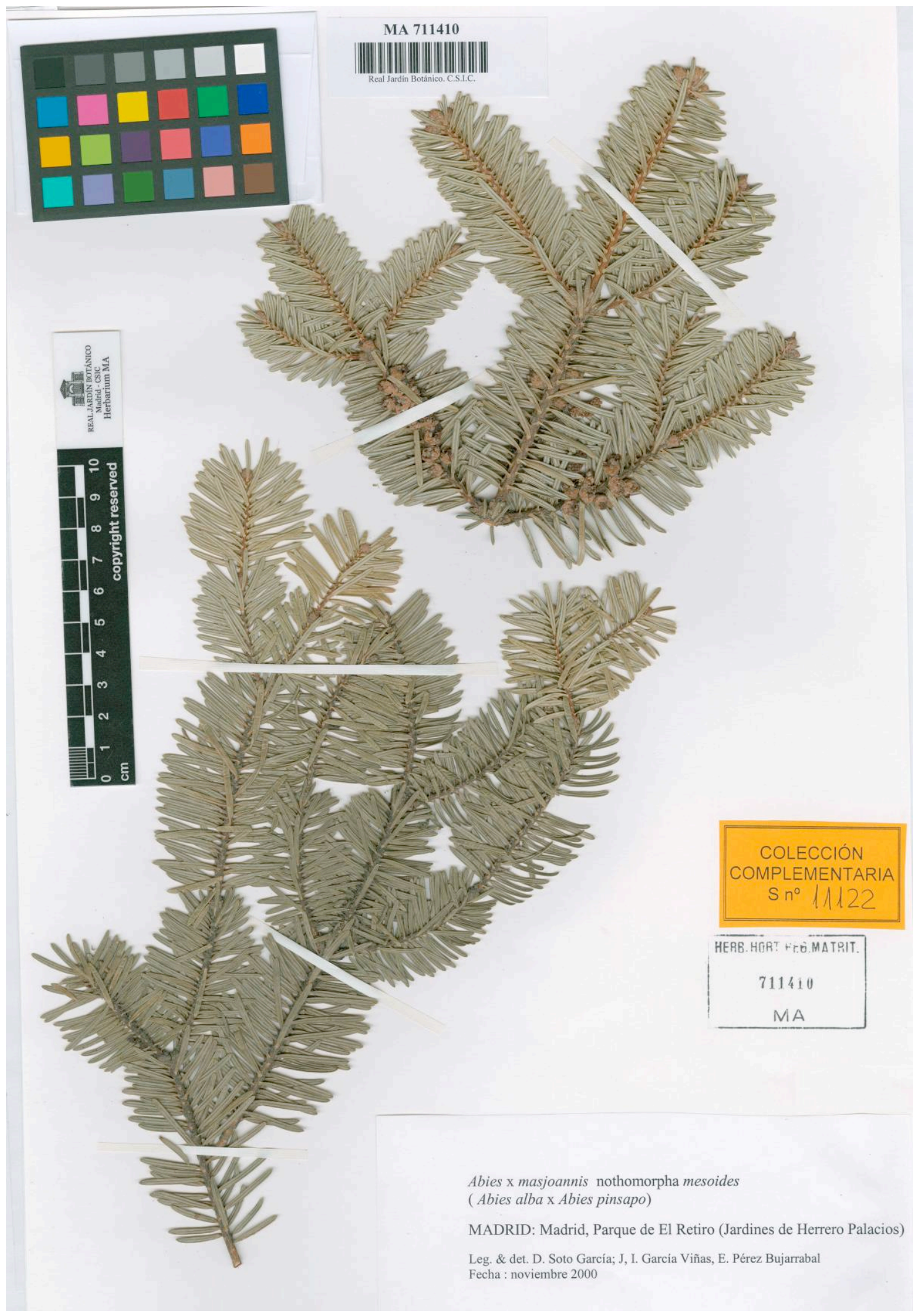

Figura 1 (Continuación). Holotipo de Abies xmasjoannis D. Soto, J.I. García Viñas et E.P. Bujarrabal [A. alba Mill. $\times$ A. pinsapo Boiss.] (MA 711410). Imagen reproducida con permiso del herbario MA. Figure 1. Holotype of Abies xmasjoannis D. Soto, J.I. Garcia Viñas et E.P. Bujarrabal [A. alba Mill. x A. pinsapo Boiss.] (MA 711410). Courtesy of the herbarium MA; reproduced with permission. 
de rango inmediatamente superior.

Para cada una de las pretendidas denominaciones: " $A$. ×masjoannis $\mathrm{nm}$. alboides", "A. xmasjoannis $\mathrm{nm}$. mesoides" y " $A$. $\times$ masjoannis $\mathrm{nm}$. pinsapoides", fue indicado como "holotipos" dos especímenes duplicados que se encuentran conservados en dos herbarios diferentes (MAIA, herbario del Departamento de Ecología del Instituto Nacional de Investigaciones Agrarias, Madrid y MA, herbario del Real Jardín Botánico de Madrid). Aunque en el trabajo de Soto García et al. (2004) se menciona suficiente material descriptivo en latín (en parte recogido en la Tabula 1) que es aplicable a la notoespecie como un todo para constituir una descripción validadora, no fue indicado ningún tipo para "Abies $\times$ masjoannis" y en consecuencia no puede ser considerado como un nombre válidamente publicado (Art. 40.1 y H. 10.1 del ICN, McNeill et al., 2012).

Por otra parte, debido a que el nombre de la especie no está publicado de forma válida, tampoco lo están los nombres de los tres pretendidos táxones infraespecíficos ("nothomorphae"); además, como "nothomorph" ya no es un rango permitido (Art. H. 12.2) no tendrían una clara indicación de rango, por lo que tampoco se habrían publicado de manera válida (Art. 37). Para validar el nombre de este híbrido, se propone lo siguiente.

\section{Resultados y discusión}

Abies $\times$ masjoannis D. Soto, J.I. García Viñas \& E.P. Bujarrabal, nothosp. nov.

[A. alba Mill. o $\times$ A. pinsapo Boiss. o]

HOLOTYPUS: España, Madrid, Parque de El
Retiro, Jardines de Herrero Palacios, cerca del antiguo foso de fieras, D. Soto García, J.I. García Viñas y E. Pérez Bujarrabal, XI-2000, MA 711410 [dos hojas]. ISOTYPUS: MAIA 5896.

DIAGNOSIS: Soto García et al. (2004: 354356, sub "Abies $\times$ masjoannis").

\section{Agradecimientos}

Al Dr. John McNeill (Royal Botanic Garden, Edinburgh) por su ayuda en el estudio del Código Internacional de Nomenclatura.

\section{Referencias}

Mcneill, J., Barrie, F.R., Buck, W.R., Demoulin, V., Greuter, W., Hawksworth, D.L., Herendeen, P.S., Knapp, S., Marhold, K., Prado, J., Prud'homme Van Reine, W.F., Smith, G.F., Wiersema, J.H. \& Turland, N.J. (eds.). (2012). International Code of Nomenclature for algae, fungi, and plants (Melbourne Code) adopted by the Eighteenth International Botanical Congress Melbourne, Australia, July 2011. Regnum Vegetabile 154. A.R.G. Gantner Verlag, Ruggell, Liechtenstein.

Ruíz De La Torre, J. (2006). Flora Mayor. Organismo Autónomo Parques Nacionales, Madrid.

Soto García, D., García Viñas, J.I. \& Pérez Bujarrabal, E. (2004). Descripción del híbrido Abies x masjoannis. Invest. Agrar.: Sist. Recur. For., 13(2), 247-356.

Soto García, D., García Viñas, J.I. \& Pérez Bujarrabal, E. (2013). Identificación rápida de Abies x masjoannis y resguardo genético de los pinsapares. En LópezQuintanilla, J., Navarro, R.M., Carreira, J.A., Coca, M. \& Rodríguez, C. (eds). Los pinsapares (Abies pinsapo Boiss.) en Andalucía: Conservación y sostenibilidad en el siglo XXI. Junta de Andalucía y Universidad de Córdoba. pp. 455-458. 